\title{
The Effects of Anisotropic Surface Roughness on Turbulent Boundary-Layer Flow
}

\author{
A. Ramani ${ }^{1}$, B. Nugroho ${ }^{1}$, A. Busse ${ }^{2}$, J. P. Monty ${ }^{1}$, N. Hutchins ${ }^{1}$, T. O. Jelly ${ }^{1,2}$ \\ ${ }^{1}$ Department of Mechanical Engineering, University of Melbourne, Victoria 3010, Australia \\ ${ }^{2}$ James Watt School of Engineering, University of Glasgow, Glasgow G12 8QQ, UK
}

\begin{abstract}
Measurements of a turbulent boundary-layer developing over systematically generated roughness are acquired for friction Reynolds numbers ranging between $3000<R e_{\tau}<6000$. A set of near-Gaussian surfaces with matched amplitude parameters and specified effective slopes in streamwise and spanwise directions are synthesised using a roughness generation algorithm. Three cases are considered: (i) an isotropic surface with equal streamwise $\left(E S_{x}=0.34\right)$ and spanwise effective slope $\left(E S_{y}=0.34\right)$; (ii) an anisotropic spanwise elongated surface with $E S_{x}=0.34$ and $E S_{y}=0.17$, and (iii) an anisotropic streamwise elongated surface with $E S_{x}=0.17$ and $E S_{y}=0.34$. The surfaces are manufactured from square sheets of acetal copolymer using an in-house CNC router. Note that surface (iii) is obtained by simply rotating surface (ii) by 90 degrees. The principal interest here is to quantify the sensitivity of the Hama roughness function to systematic changes in surface anisotropy. To this end, hot-wire anemometry measurements are acquired at three different freestream velocities under zero-pressure gradient conditions for each surface. Relative to the isotropic case, an increase in the turbulence intensity is seen in the near-wall region for the anisotropic cases. As expected, decreasing $E S_{x}$ leads to a lower mean momentum deficit which confirms the findings of many previous experimental and numerical studies. However, results also suggest that $E S_{y}$ plays an important role. Even for the mildly anisotropic case considered here, the roughness function is seen to vary by up to $15 \%$ as $E S_{y}$ is reduced while $E S_{x}$ is held constant. In addition, regions of high streamwise dispersive velocity are seen to extend further into the flow field as $E S_{y}$ reduces. These observations suggest that existing models for drag prediction need to be modified to account for surface anisotropy.
\end{abstract}

\section{Keywords}

Turbulence; Boundary layers; Roughness

\section{Introduction}

Wall-bounded turbulence in engineering and atmospheric flows often occurs over rough surfaces. In most cases, the presence of roughness induces additional drag which is of tremendous practical concern. For example, roughness due to biofouling on a ship hull can lead to substantial increases in skin friction drag and hence fuel burn. The economic impact of this additional fuel burn is estimated to be $\$ 1$ billion over 15 years for just a single class of US Navy vessels (just 56 vessels from the estimated 90,000 operating worldwide) [14]. Physically, this increase in drag is quantified by a downward shift of the innerscaled mean velocity profile by the quantity $\Delta U^{+}$, also known as the Hama roughness function. A wide variety of roughness topographies, both naturally occurring and man-made, can be observed on surfaces of practical interest. An enduring goal in fluid dynamics research is to correlate key topographical parameters with the Hama roughness function. A common currency used to compare the various topographies is the equivalent sandgrain roughness $\left(k_{s}\right)$.
In the fully-rough regime, the roughness function can then be evaluated as:

$$
\Delta U^{+}=\kappa^{-1} \log k_{s}^{+}+A-8.5
$$

where $\kappa=0.384$ is the von Kármán constant and $A=4.17$ is the smooth wall intercept. Tools such as the Moody chart use $k_{s}$ to determine the drag. However, $k_{s}$ is a hydrodynamic quantity and cannot be measured directly from the surface (e.g. a scan). Currently, it must be determined from measurements of the flow which is an expensive process. Therefore, correlations for predicting $k_{s}$ based on readily measurable topography quantities are highly valuable. This is not a straightforward task as many different parameters exist for characterising realistic, three-dimensional and multi-scale roughness. Many correlations have been proposed based on key topographical parameters identified thus far. These include the root mean square height $\left(S_{q}\right)$, skewness $\left(S_{s k}\right)$ [6] [7], and streamwise effective slope $\left(E S_{x}\right)$ [12] [4]. The latter quantity is defined as the mean absolute gradient of the surface in the direction of the flow. It is given as $E S_{x}=(1 / A) \int|\partial h(x, y) / \partial x| d A$ where $h(x, y)$ is the heightmap of the roughness and $A$ is the planform area. These correlations, which have considered both regular and irregular geometries, work well for the cases from which they are derived. However, they do not account for spanwise anisotropy, which can be quantified by introducing the spanwise effective slope, $E S_{y}$, as an additional parameter. $E S_{y}$ is defined analogously and is given as $E S_{y}=(1 / A) \int|\partial h(x, y) / \partial y| d A$. Considering that many forms of natural and artificial roughness have some degree of anisotropy [1], the consideration of $E S_{y}$ in addition to $E S_{x}$ seems important.

The case of isotropic roughness, where $E S_{x}=E S_{y}$, has been studied and extensively characterised for both regular [4] and irregular geometries [9]. Recent studies have shown that spanwise anisotropy strongly affects the near-wall region of the flow [2]. However, studies on the effect of anisotropy where the ratio $E S_{x} / E S_{y}$ is systematically varied remain scarce. One reason for this scarcity is that studies with finite $E S_{x}$ and $E S_{y}$ are generally based on scans of real surfaces where $E S_{x}$ and $E S_{y}$ cannot be systematically varied. This can be overcome by synthetically generating the rough surfaces with specified slopes. The present study aims to be the first to experimentally characterise the effects of surface anisotropy by systematically varying $E S_{x} / E S_{y}$.

\section{Experiment Setup}

Irregular, three-dimensional heightmaps are synthesised using a surface generation algorithm [8]. The heightmaps are generated on a $300 \mathrm{~mm} \times 300 \mathrm{~mm}$ computational tile with doubly-periodic boundaries. The surfaces generated are near-Gaussian with zero mean and share a common mean peak-to-valley height $\left(S_{z, 5 \times 5}\right)$, which is the average height difference between the highest peaks and the lowest valleys [3]. Therefore, the surfaces have identical amplitude parameters and the effects of anisotropy are isolated from higher order moments such as skewness. The values of $E S_{x}$ and $E S_{y}$ are chosen based on the limits of the wavy 

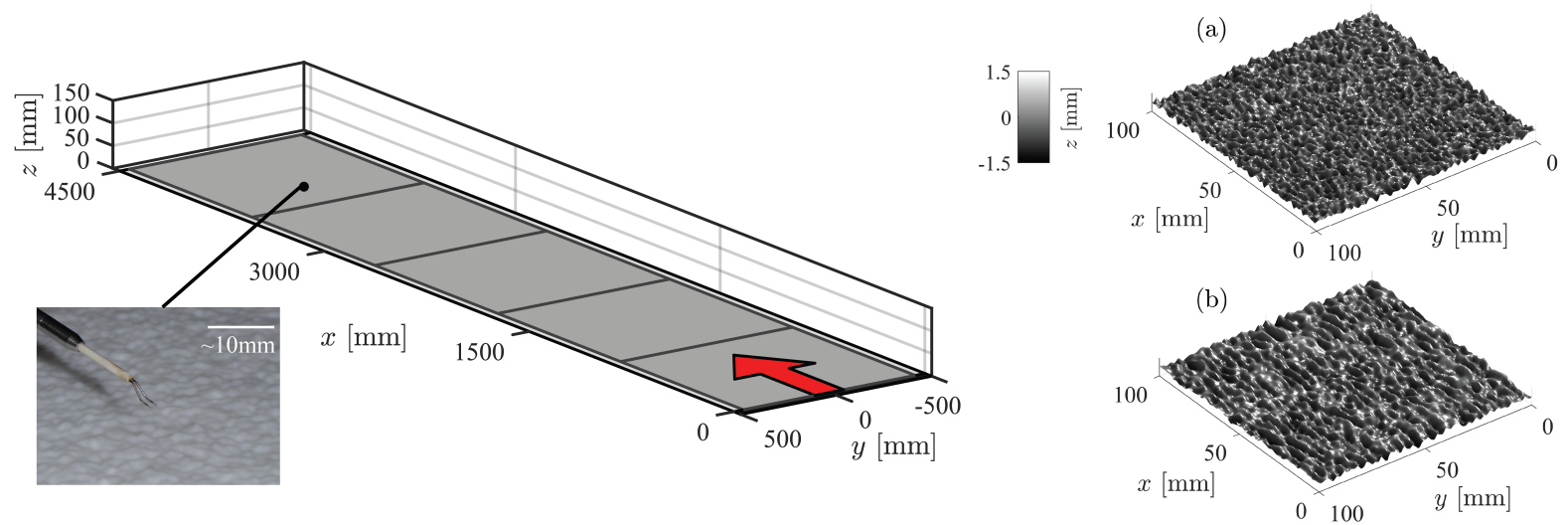

Figure 1. Left: Schematic of the rough-wall experiment setup. The hot-wire measurements are carried out at a streamwise position of $x \approx 3.8 \mathrm{~m}$ from the trip at the inlet of the tunnel. A close-up photograph of the surface and the hot-wire probe is shown in the inset. The flow direction is indicated by the red arrow. Right: Illustrative heightmaps generated for the surfaces in this study. (a) Case 034_034 for isotropic roughness; (b) Case 017_034 with streamwise elongated features. Note: The $z$ axis is scaled $2: 1$ to enhance clarity.

regime ( $E S_{x} \approx 0.35$ ) [6] [12] and the limit at which fully rough behaviour is expected $\left(E S_{x} \approx 0.12\right)$ [12]. The surface statistics are summarised in Table 1. The illustrative heightmaps are shown in Figure 1 (a) and (b).

\begin{tabular}{|c|c|c|c|c|}
\hline Case & $E S_{x}$ & $E S_{y}$ & $\begin{array}{c}U_{\infty} \\
{[\mathrm{m} / \mathrm{s}]}\end{array}$ & $\begin{array}{c}\text { Line/ } \\
\text { Symbol }\end{array}$ \\
\hline 034_034 & 0.345 & 0.344 & 15.0 & $-\bullet$ \\
$034 \_034$ & 0.345 & 0.344 & 20.0 & -- \\
$034 \_034$ & 0.345 & 0.344 & 25.0 & - \\
\hline $034 \_017$ & 0.346 & 0.167 & 14.9 & $-\bullet$ \\
$034 \_017$ & 0.346 & 0.167 & 20.0 & -- \\
$034 \_017$ & 0.346 & 0.167 & 25.0 & - \\
\hline $017 \_034$ & 0.167 & 0.346 & 15.0 & - \\
017_034 & 0.167 & 0.346 & 19.9 & -- \\
017_034 & 0.167 & 0.346 & 25.0 & - \\
\hline
\end{tabular}

Table 1. Summary of the roughness cases and the measurement free-stream velocities. For all cases, $\left(S_{z, 5 \times 5}, S_{q}\right)=(3.0,0.4) \mathrm{mm}$ and $\left(S_{s k}, S_{k u}\right) \approx(0,3)$.

The surfaces are machined from acetal co-polymer sheets using an in-house CNC router. Two sets of surfaces are manufactured. For Case 034_034, five tiles spanning $900 \mathrm{~mm} \times 900 \mathrm{~mm}$ each are produced. The physical tiles therefore contain a $3 \times 3$ array of the numerically generated heightmap. The finishing pass is carried out with a $1.5 \mathrm{~mm}$ diameter ball-nosed cutter with a step-over distance of $0.25 \mathrm{~mm}$ which gives a surface finish accuracy of less than $10 \mu \mathrm{m}$ (which, for the highest Reynolds number in this study, corresponds to a viscous length-scale of $z^{+}<1$ where $z^{+}=z U_{\tau} / v$ ). For the anisotropic cases, only two $900 \mathrm{~mm} \times 900 \mathrm{~mm}$ tiles are manufactured to be placed immediately upstream of the measurement station. This corresponds to a fetch of about $25 \delta$ (where $\delta$, the $99 \%$ boundary-layer thickness, is based on the isotropic case) which is sufficient for the boundary-layer to recover to the new wall conditions [10]. The finishing pass for this surface is carried out with a $2 \mathrm{~mm}$ diameter ball-nosed cutter with a step-over distance of $0.6 \mathrm{~mm}$. This limits the surface finish accuracy to about $40 \mu \mathrm{m}$ (or $z^{+}<3$ ). These allowances are made due to facility usage restrictions and are deemed acceptable for the purposes of this study. A closeup photograph of the machined surface from Case 034_034 is shown in the inset in Figure 1. Note that Case 017_034 is set-up by simply rotating Case $034 \_017$ by 90 degrees.

Measurements are carried out in a zero-pressure gradient, open- return type boundary-layer wind-tunnel (see [13] for further details). The working section of the tunnel has dimensions of $6.7 \mathrm{~m} \times 0.94 \mathrm{~m} \times 0.38 \mathrm{~m}$. The measurement station is set up at a streamwise position of $x \approx 3.8 \mathrm{~m}$ from the trip at the inlet (see Figure 1). A modified DANTEC 55P05 probe with $5 \mu \mathrm{m}$ platinum core Wollaston wire is used. The probe is manufactured in-house and the length $(l)$ to diameter $(d)$ ratio of the sensing element is set to $l / d \approx 200$ in order to minimise attenuation due to end-conduction effects. The probe is operated in the constant temperature mode with an overheat ratio of 1.8 using an in-house circuit. Measurements are made over a grid in the $y-z$ (spanwise wall-normal) plane which spans $y \approx \pm 1 \delta$ and $z \approx 1.6 \delta$. Sampling duration is set to ensure convergence of second order statistics. Note that the same probe is used for a range of Reynolds numbers therefore measurements at higher velocities will experience attenuation due to insufficient spatial resolution. A temperature based interpolation scheme is used to account for the calibration drift. Calibration is performed at the start and end of each measurement and at the end of each wall-normal traverse in the free-stream [16].

A first estimate of friction velocity $\left(U_{\tau}\right)$ and virtual origin $(\varepsilon)$ is obtained by the modified Clauser method. However, the uncertainty associated with this method can be $\pm 4 \%$ [15]. Therefore, an additional estimate of friction velocity is obtained from the mean velocity defect and turbulence intensity profiles in the outer region $(0.2<z / \delta<1)$. By assuming Townsend's outerlayer similarity holds, $U_{\tau}$ can be obtained by simultaneously minimising the combined differences between the rough and smooth wall data [11]. The two methods yield estimates which are within $7 \%$ of each other. A further validation of the measurements is obtained by evaluating the momentum thickness $(\theta)$ for each case as a measure of the integrated skin-friction coefficient which is seen to confirm the salient trends.

\section{Results and Discussion}

The mean velocity defect and the mean turbulence intensity profiles are shown in Figure 2 (a) and (b) respectively. Note that the value of $U_{\tau}$ used here is the one estimated by simultaneously minimising the difference between the smooth and rough wall data in the outer region, $z / \delta>0.2$, for both the defect and turbulence intensity curves. While this assumes outer-layer similarity, the simultaneous collapse between the rough and smooth results in both profiles suggests that this is a reasonable assumption. Closer to the wall $(z / \delta<0.1)$, an increase in the turbulence intensity is seen for the anisotropic cases compared to 

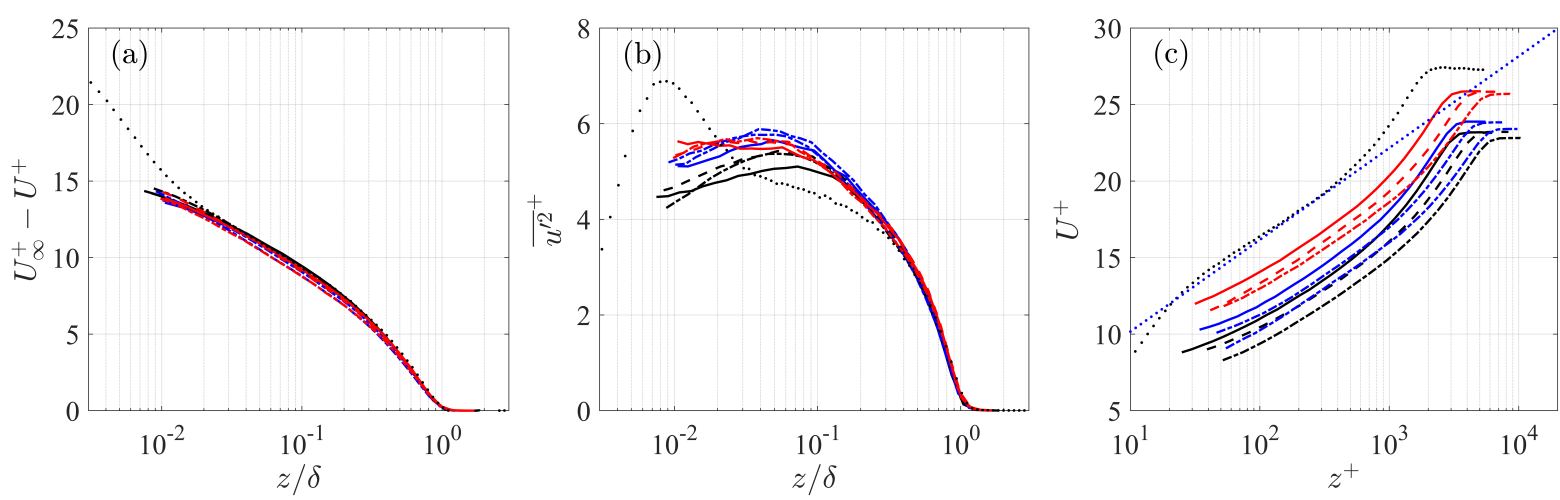

Figure 2. (a) Mean velocity defect; (b) mean turbulence intensity profiles with outer scaling and (c) inner-scaled mean velocity profiles. The dotted black line is a reference smooth wall profile. In (c), the dotted blue line is the smooth wall $\log \operatorname{law} U^{+}=\kappa^{-1} \log z^{+}+A$. See Table 1 for line types.

\begin{tabular}{|c|c|c|c|c|c|c|c|}
\hline Case & $\begin{array}{c}U_{\infty} \\
{[\mathrm{m} / \mathrm{s}]}\end{array}$ & $\begin{array}{c}U_{\tau} \\
{[\mathrm{m} / \mathrm{s}]}\end{array}$ & $\Delta U^{+}$ & $k_{s}^{+}$ & $\delta^{+}$ & $\begin{array}{c}\theta \\
{[\mathrm{mm}]}\end{array}$ & $\begin{array}{c}C_{f} \times \\
10^{-3}\end{array}$ \\
\hline 034_034 & 15.0 & 0.66 & 5.5 & 43.1 & 3410 & 10.9 & 3.73 \\
$034 \_034$ & 20.0 & 0.88 & 6.2 & 56.3 & 4708 & 11.2 & 3.71 \\
$034 \_034$ & 25.0 & 1.12 & 7.2 & 82.3 & 5828 & 11.5 & 3.84 \\
\hline $034 \_017$ & 14.9 & 0.62 & 4.5 & 29.8 & 3245 & 10.1 & 3.51 \\
$034 \_017$ & 20.0 & 0.84 & 5.2 & 39.4 & 4418 & 10.2 & 3.52 \\
$034 \_017$ & 25.0 & 1.07 & 6.1 & 55.5 & 5633 & 10.5 & 3.65 \\
\hline $017 \_034$ & 15.0 & 0.58 & 2.3 & 12.8 & 2961 & 9.5 & 2.99 \\
$017 \_034$ & 19.9 & 0.77 & 3.0 & 16.8 & 3919 & 9.7 & 2.99 \\
017_034 & 25.0 & 0.97 & 3.5 & 20.2 & 4966 & 9.8 & 3.03 \\
\hline
\end{tabular}

Table 2. Mean flow quantities. The superscript + indicates inner scaling, i.e. $\delta^{+}=\delta U_{\tau} / \mathrm{v}$, where $\delta$ is the $99 \%$ boundary-layer thickness.

the isotropic case beyond the expected increases due to increasing Reynolds number. This suggests that spanwise anisotropy, when $E S_{x} / E S_{y} \neq 1$, influences the near-wall turbulence.

The mean flow quantities are summarised in Table 2. The innerscaled mean velocity profiles are shown in Figure 2 (c). As expected, the velocity profiles display a downward shift, $\Delta U^{+}$, from the reference smooth wall profile which increases with increasing Reynolds number. In Figure $3, \Delta U^{+}$is plotted as a function of $E S_{x}$ and $E S_{y}$ in plots (a) and (b) respectively. When $E S_{y}$ is held constant and $E S_{x}$ is reduced, $\Delta U^{+}$is observed to drop by about $50 \%$. This underscores the importance of $E S_{x}$ as a key parameter for drag prediction. However, when $E S_{x}$ is held constant and $E S_{y}$ is reduced, $\Delta U^{+}$is observed to reduce by $15-18 \%$. This is beyond the uncertainty in $\Delta U^{+}$estimates of $\pm 10 \%$ [15]. This suggests that $E S_{y}$ also has an appreciable effect on the overall drag even for the mildly anisotropic case considered here. The value of $C_{f}=2\left(U_{\tau} / U_{\infty}\right)^{2}$, shown in Table 2, varies less than $4 \%$ across the Reynolds number range for all cases which suggests that fully rough conditions have been reached. Therefore, $k_{s}^{+}$can be evaluated using Eq. 1 and is shown in Table 2. These results suggest that the current irregular rough surfaces approach the fully rough behaviour even at low values of $\Delta U^{+}$. It is also evident that the total drag on an anisotropic rough surface is strongly dependent on the flow direction. Therefore, empirical correlations for the prediction of the roughness function must factor in the surface anisotropy, i.e. include both $E S_{x}$ and $E S_{y}$, for more accurate drag prediction.

The effects of varying $E S_{x}$ and $E S_{y}$ can also be assessed from the streamwise dispersive velocities in the spanwise wallnormal plane, defined here as the difference between the localtime averaged velocity and the span- and time-averaged veloc-
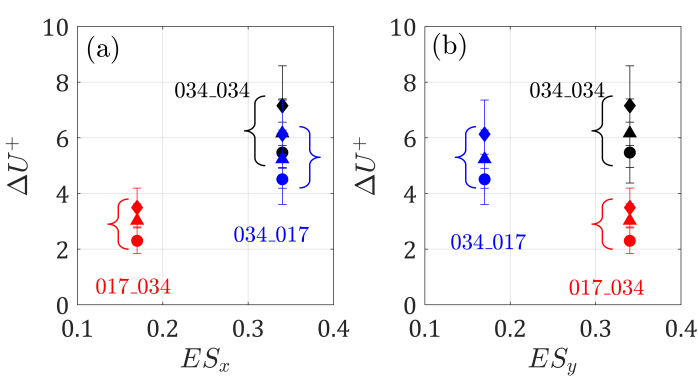

Figure 3. $\Delta U^{+}$as a function of (a) $E S_{x}$ and (b) $E S_{y}$ (with error bars for $\pm 10 \%$ uncertainty). The braces indicate the values for the same roughness cases at different Reynolds numbers. See Table 1 for symbols.

\begin{tabular}{|c|c|c|}
\hline Case & $\bar{\lambda}_{x} / \delta$ & $\bar{\lambda}_{y} / \delta$ \\
\hline $034 \_034$ & 0.138 & 0.138 \\
$034 \_017$ & 0.154 & 0.294 \\
$017 \_034$ & 0.294 & 0.154 \\
\hline
\end{tabular}

Table 3. Mean wavelengths of the roughness, where $\bar{\lambda} / 2 \pi=m_{0} / m_{1}$ and $m_{n}$ is the $n$th spectral moment.

ity, i.e. $\tilde{U}(y, z)=U(y, z)-<U(z)>$ [3]. In Figure 4, contours of $\tilde{U}(y, z)$ are shown for each of the cases at $U_{\infty} \approx 25 \mathrm{~m} / \mathrm{s}$. In the outer region with $z>0.2 \delta, \tilde{U}(y, z)$ varies less than $\pm 1 \%$ which suggests spanwise homogeneity of the flow.

On the other hand, strong variations up to $\pm 10 \%$ are seen in the near-wall region for all the cases, which reflects the wall-normal extent of the roughness sublayer [3]. As seen in Figure 4 (b), the roughness sublayer appears to extend furthest into the flow for the 034_017 case compared to the 034_034 and 017_034 cases. To further understand this, the mean in-plane wavelengths $(\bar{\lambda})$ of the surface, obtained from the ratio of spectral moments [17], are compared in Table 3 . When $\bar{\lambda}_{y} / \delta \approx 1$, streamwise rollmodes are induced that span the entire boundary-layer [5]. For $\bar{\lambda}_{y} / \delta<0.4$, the roll modes are confined to the near wall region and spanwise homogeneity can be expected further from the surface [5]. In all the cases considered here, $\bar{\lambda}_{y} / \delta<0.4$. Therefore, spanwise homogeneity is observed beyond a nearwall roughness sublayer. Recent results have suggested that the height of the roughness sublayer is proportional to $\bar{\lambda}_{y}$ and extends to $z / \bar{\lambda}_{y} \approx 0.5$ [4]. This approximate limit is marked by the dashed line in Figure 4 and seems to reasonably bound, in all cases, the region of strong dispersive variations near the wall. 
(a)

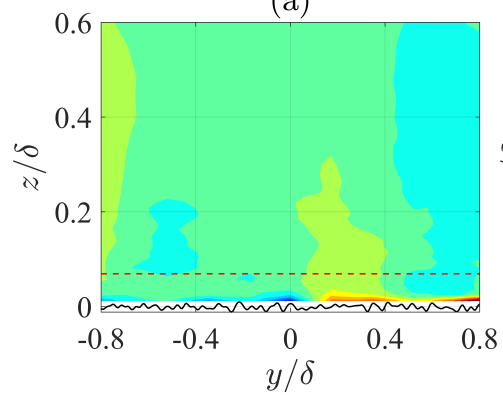

(b)

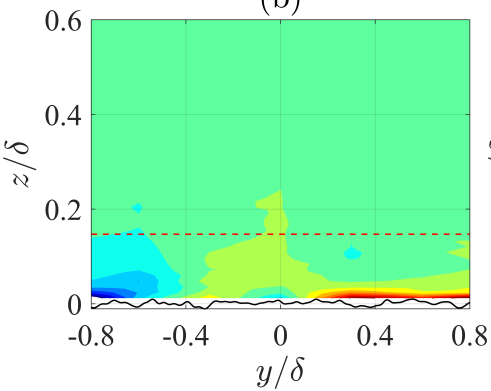

(c)

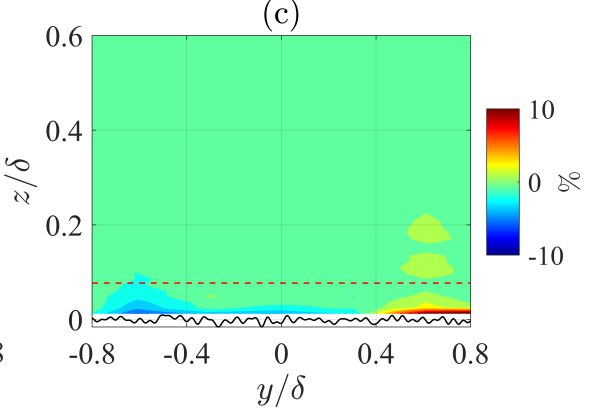

Figure 4. Contours of streamwise dispersive velocities, $\tilde{U}(y, z)$, as a percentage of the spanwise averaged mean velocity, $100 \times \tilde{U}(y, z) /<U(z)>$ at $U_{\infty}=25 \mathrm{~m} / \mathrm{s}$ for (a) 034_034, (b) 034_017 and (c) 017_034. The black line represents the roughness profile. The red dashed line indicates the extents of the roughness sublayer at $z / \bar{\lambda}_{y} \approx 0.5$.

\section{Conclusions}

The effect of surface roughness anisotropy on turbulent boundary-layer flow is systematically examined by varying the ratio of streamwise and spanwise effective slope. Three cases are considered - an isotropic surface with $E S_{x}=E S_{y}=0.34$, and two anisotropic cases with $E S_{x}=0.34$ and $E S_{y}=0.17$ and vice versa. Amplitude parameters are matched for all cases. Hence, the cases only differ in their streamwise and spanwise effective slopes. Hot-wire measurements are performed to assess the impact on the flow. For both anisotropic cases, an increase in the near-wall turbulence intensity is seen compared to the isotropic case. The roughness function is seen to vary by about $50 \%$ between the highest and lowest tested values of $E S_{x}$ which confirms the findings in literature that it is an important parameter for drag prediction. However, the roughness function is also seen to reduce by as much as $18 \%$ as $E S_{y}$ is varied with $E S_{x}$ held constant. Further, the region of higher dispersive velocity near the wall is seen to extend further into the flow field as $E S$ r reduces and the spanwise characteristic wavelength increases. These results suggest that accurate drag prediction correlations must therefore account for surface anisotropy when $E S_{x} / E S_{y} \neq 1$.

\section{Acknowledgements}

The authors gratefully acknowledge the financial support of the University of Melbourne Early Career Researcher grant and the Australian Research Council.

\section{References}

[1] Bons, J., Taylor, R., McClain, S. and Rivir, R., The many faces of turbine surface roughness, J. Turbomach., 123, 2001, 739-748.

[2] Busse, A. and Jelly, T., Influence of surface anisotropy on turbulent flow over irregular roughness, Flow Turbul. Combust., 104, 2020, 331-354.

[3] Busse, A., Thakkar, M. and Sandham, N., Reynoldsnumber dependence of the near-wall flow over irregular rough surfaces, J. Fluid Mech., 810, 2017, 196-224.

[4] Chan, L., MacDonald, M., Chung, D., Hutchins, N. and Ooi, A., Secondary motion in turbulent pipe flow with three-dimensional roughness, J. Fluid Mech., 854, 2018, 5-33.

[5] Chung, D., Monty, J. and Hutchins, N., Similarity and structure of wall turbulence with lateral wall shear stress variations, J. Fluid Mech., 847, 2018, 591-613.
[6] Flack, K. and Schultz, M., Roughness effects on wallbounded turbulent flows, Phys. Fluids, 26, 2014, 101305.

[7] Jelly, T. and Busse, A., Reynolds and dispersive shear stress contributions above highly skewed roughness, $J$. Fluid Mech., 852, 2018, 710-724.

[8] Jelly, T. and Busse, A., Multi-scale anisotropic rough surface algorithm: Technical documentation and user guide, Technical report, University of Glasgow, 2019.

[9] Jelly, T. and Busse, A., Reynolds number dependence of reynolds and dispersive stresses in turbulent channel flow past irregular near-Gaussian roughness, Int. J. Heat Fluid Fl., 80, 2019, 108485.

[10] Li, M., de Silva, C., Rouhi, A., Baidya, R., Chung, D., Marusic, I. and Hutchins, N., Recovery of wall-shear stress to equilibrium flow conditions after a rough-tosmooth step change in turbulent boundary layers, J. Fluid Mech., 872, 2019, 472-491.

[11] Monty, J., Dogan, E., Hanson, R., Scardino, A., Ganapathisubramani, B. and Hutchins, N., An assessment of the ship drag penalty arising from light calcareous tubeworm fouling, Biofouling, 32, 2016, 451-464.

[12] Napoli, E., Armenio, V. and De Marchis, M., The effect of the slope of irregularly distributed roughness elements on turbulent wall-bounded flows, J. Fluid Mech., 613, 2008, 385-394.

[13] Nugroho, B., Hutchins, N. and Monty, J., Large-scale spanwise periodicity in a turbulent boundary layer induced by highly ordered and directional surface roughness, Int. J. Heat Fluid Fl., 41, 2013, 90-102.

[14] Schultz, M., Bendick, J., Holm, E. and Hertel, W., Economic impact of biofouling on a naval surface ship, Biofouling, 27, 2011, 87-98.

[15] Schultz, M. and Flack, K., The rough-wall turbulent boundary layer from the hydraulically smooth to the fully rough regime, J. Fluid Mech., 580, 2007, 381-405.

[16] Talluru, K., Kulandaivelu, V., Hutchins, N. and Marusic, I., A calibration technique to correct sensor drift issues in hot-wire anemometry, Meas. Sci. Technol., 25, 2014, 105304.

[17] Townsin, R., The ship hull fouling penalty, Biofouling, 19, 2003, 9-15. 